\title{
Association of Bovine Papillomavirus Type 1 E6 oncoprotein with the focal adhesion protein paxillin through a conserved protein interaction motif
}

\author{
Scott B Vande Pol $^{1}$, Michael C Brown ${ }^{2}$ and Christopher E Turner ${ }^{2}$ \\ ${ }^{1}$ Case Western Reserve University School of Medicine, Institute of Pathology; ${ }^{2}$ Department of Anatomy and Cell Biology, State \\ University of New York at Syracuse, USA
}

\begin{abstract}
We have found that the E6 oncoprotein of Bovine Papillomavirus Type 1 (BE6) as well as the E6 protein of the cancer associated HPV-16 (16E6) interact with the focal adhesion protein paxillin. Mutational analysis of paxillin revealed that BE6 binds paxillin through small protein interaction motifs called LD motifs that have been previously identified as important in regulating association of paxillin with vinculin and focal adhesion kinase (FAK), and that BE6 can interact with at least two separate binding sites on paxillin. The LD motifs of paxillin that bind BE6 share homology with the E6 binding site of E6-AP, a ubiquitin ligase that together with 16E6 targets the degradation of the p53 tumor suppressor. Paxillin binding to BE6 excludes simultaneous binding to E6-AP. Mutational analysis of BE6 can distinguish the interaction of BE6 with E6-AP compared to paxillin and revealed that the interaction of BE6 with paxillin may be necessary for the induction of anchorage independent growth of cells by BE6.
\end{abstract}

Keywords: integrin; transformation; cytoskeleton; phosphotyrosine

\section{Introduction}

The papillomavirus E6 oncoproteins are small zincbinding proteins that have been shown to function as adaptor proteins in altering the metabolism of the $\mathrm{p} 53$ tumor suppressor. E6 proteins from the cancerassociated human papillomaviruses (HPVs) complex with a cellular protein termed E6-AP, and together with E6-AP bind to the p53 tumor suppressor protein (Huibregtse et al., 1991). Formation of the E6-E6APp53 complex leads to the degradation of p53 through ubiquitin-mediated proteolysis in vitro (Huibregtse et al., 1993a). E6 interacts with a 18 amino acid sequence in E6-AP and functions as an adaptor protein in the complex with p53, inasmuch as E6-AP does not interact with p53 in the absence of E6 and the degradation of p53 requires both E6 and E6-AP (Huibregtse et al., 1993b).

E6 genes from the non-cancer associated HPV types have shown surprising biochemical diversity compared to the cancer associated HPVs in that they have not been shown to bind E6-AP or degrade p53. Similarly, BPV-1 E6 (BE6), while binding E6-AP, fails to either complex with p53 or degrade other associated proteins in an in vitro degradation assay, implying that BE6

Correspondence: SB Vande Pol

Received 8 May 1997; revised 15 August 1997; accepted 15 August 1997 association with E6-AP may have functions other than the targeting of p53 (Chen et al., 1995; Scheffner et al., 1992).

The targeted degradation of p53 by cancer associated HPV-16 E6 is associated with the immortalization of primary breast epithelial cells by E6 (Dalal et al., 1996) and the abrogation of cell cycle checkpoints by E6, but may not be related to the ability of E6 oncoproteins to induce anchorage independent growth. This is illustrated by E6 genes that do not target p53, such as BE6 and non-cancer associated HPV types that can induce anchorage independent growth in contact inhibited rodent cell lines, implying that the E6 oncoproteins have transforming functions independent of E6-AP or p53 association (Schiller et al., 1984; Trujilo and Mounts, 1996; Yang et al., 1985). Recently, the cancer associated HPV E6 proteins as well as BE6 have been shown to associate with a calcium-binding protein localized to the endoplasmic reticulum, called ERC-55 or E6-BP. A limited mutational analysis indicated that association of BE6 with ERC-55 correlated with transformation by BE6, yet the function of ERC-55 and the role it may play in transformation by BE6 is as yet undetermined (Chen et al., 1995).

When anchorage dependent cells attach to substratum, a partly characterized series of events unfolds: cellular integrin molecules engage extracellular matrix components, resulting in tyrosine phosphorylation of proteins including pp125 focal adhesion kinase (FAK) and paxillin; this phosphorylation event is accompanied by the re-organization of actin filaments into stress fibers that terminate in discrete sites of adhesion between the membrane and the substratum, called focal adhesions (reviewed in Turner, 1994). The parallel tyrosine phosphorylation of FAK and paxillin, together with the reorganization of the actin cytoskeleton and the localization of FAK and paxillin in focal adhesions suggests that FAK and paxillin may be instrumental in the reorganization of the cytoskeleton in response to cell attachment.

How paxillin contributes to this process is unknown, but several features of paxillin suggest that it may play a regulatory rather than structural role in the remodeling of the cytoskeleton. As would be expected for a regulatory protein, paxillin is considerably less abundant than structural cytoskeletal proteins such as talin or vinculin, yet paxillin directly associates with vinculin (Turner et al., 1990; Wood et al., 1994). Paxillin contains features of a regulatory protein, including $\mathrm{SH} 2$-binding and proline rich SH3-binding motifs (Salgia et al., 1995a; Turner and Miller, 1994). Paxillin associates with or has been identified as a substrate of a number of provocative kinases and 
oncogenes including the non-receptor kinases v-src (paxillin was first identified as a major tyrosine phosphorylated protein in v-src transformed cells (Glenney and Zokas, 1989)), c-src (Weng et al., 1993), Csk (c-terminal src kinase) (Sabe et al., 1994), FAK (focal adhesion kinase) (Bellis et al., 1995; Hildebrand et al., 1995; Tachibana et al., 1995; Turner and Miller, 1994), the adapter protein v-crk (Birge et al., 1993), and to BCR/ABL through its association with Crkl (Salgia et al., 1995b). Phosphorylation of paxillin by FAK creates a $\mathrm{SH} 2$ binding motif that interacts with Crk (Schaller and Parsons, 1995). It has recently been shown that paxillin directly interacts with FAK and vinculin through short leucine rich peptide motifs termed LD motifs; there are four such motifs in paxillin, two of which can interact with FAK and vinculin (Brown et al., 1996). The c-terminal portion of paxillin contains four LIM domains, one of which (LIM domain three) targets paxillin to focal adhesions through interactions with unidentified proteins (Brown et al., 1996).

While paxillin is associated with proteins and events implicated in oncogenesis and re-modeling of the cytoskeleton, its role in these events is as yet undetermined. In $\mathrm{v}-s r c$ transformed fibroblasts there is loss of normal cell adhesion and cell spreading, v-src is localized to focal adhesions, paxillin and FAK are hyper-phosphorylated, and there is disruption of normal cytoskeletal focal adhesions. In contrast, when adherent cells bind to substratum, FAK and paxillin phosphorylation are associated with adherence to the substratum, cell spreading, and formation of focal adhesions. It is as yet unclear how to reconcile the role of paxillin in these two disparate observations.

In order to better understand the role of E6 oncoproteins in cell transformation, we sought to identify potential targets of the BE6 oncoprotein by performing a yeast two-hybrid search using a BE6 bait. We identified the focal adhesion protein paxillin as an interactor with both BE6 and the cancer-associated HPV-16 E6 (16E6). BE6 interacts with paxillin through at least 2 LD peptide motifs. The LD motifs of paxillin that interact with BE6 are homologous to the peptide motif of E6-AP that interacts with BE6. This identifies a small conserved protein interaction motif that interacts with E6 adaptor proteins, and indicates that LD domains may have a general role in mediating protein-protein interactions. An analysis of BE6 mutants is consistent with a role for the BE6-paxillin interaction in the induction of anchorage independent growth by BE6.

\section{Results}

\section{Paxillin interacts with E6 oncoproteins}

In order to identify proteins that interact with BE6 we performed a 2-hybrid interaction hunt. $1.4 \times 10^{6}$ human cDNAs were screened for interaction with a lexA-BE6 bait plasmid and failure to interact with lexA-BE7 (lexA fused to the BPV-1 E7 protein). BE7 was used as a non-specific bait in this assay as it contains a zincbinding motif similar in structure to the two zincbinding motifs of BE6. One colony that interacted with BE6 but not BE7 was determined by $5^{\prime}$ and $3^{\prime}$ sequencing of the cDNA insert to contain a cDNA encoding for the first 463 amino acids of paxillin, with the cDNA extending five amino acids amino terminal of the initiating methionine. A full-length cDNA clone for chicken paxillin was expressed as a fusion to the B42 transactivation domain and tested for association with either BE6 or other non-BE6 lexA fusions in a 2hybrid yeast interaction assay. Figure la shows that both paxillin and the E6-interacting protein E6-AP strongly interacted with BE6 in yeast. Paxillin did not interact with control lexA fusions including other zinc containing proteins (lexA-BE7, or lexA fused to the regulatory domain of casein kinase II). Although not visible in the Figure, longer incubation of the XGAL plate shown in Figure 1a did reveal a weak interaction between paxillin and HPV-16 E6 (16E6), that was confirmed in an in vitro interaction assay (Figure 1b).

The interaction between BE6 and paxillin observed in yeast was confirmed in an in vitro interaction assay shown in Figure 1b. The indicated proteins were expressed in vitro (Figure 1b upper panel) and tested for their ability to bind to a maltose-binding-protein/ paxillin fusion (MBP-paxillin) immobilized on amyloseagarose beads (Figure 1b lower panel). Only BE6 and $16 \mathrm{E} 6$ were retained on the MBP-paxillin beads. Nonbinding control proteins included the LIM-domain protein trip6 (Lee et al., 1995) zinc-binding proteins (p53, E7 from BPV-1 (BE7)), the adaptor protein 14-33 , and the E6-interacting protein E6-AP.

In order to determine if BE6 could interact with paxillin in mammalian cells, GST-BE6 or GST-BE7 was transiently overexpressed in CV-1 cells, the cells were lysed and the cell lysates incubated with glutathione agarose beads. Expression of GST-BE6 or GST-BE7 did not alter the amount or the mobility of paxillin by Western blot analysis (Figure 1c lanes 1-4). GST-BE6 retained paxillin on the glutathione-agarose beads while GST-BE7 did not (Figure 1c lanes 7 and 8). In experiments not shown here, GST-BE6 induces anchorage independent growth when expressed in mouse $\mathrm{C} 127$ cells (Vande Pol, S, unpublished observations). The blot shown in Figure 1c was subsequently reprobed with a rabbit antibody to GST, confirming similar levels of expression of GST-BE6 and GST-BE7 (data not shown).

Figure 1 demonstrates that native paxillin interacts with both lexA and GST fusions of BE6 in vitro and in vivo (Figures 1a and $\mathrm{c}$ ), and that native BE6 and 16E6 interact with paxillin fusions in vitro (Figure 1b). In order to determine if mammalian or yeast proteins are required to facilitate the BE6-paxillin interaction, MBP, MBP-paxillin, GST, or GST-BE6 were expressed in bacteria and the clarified but unpurified bacterial extracts were tested for the ability of BE6 and paxillin to interact in vitro. Figure 2 lanes 1-3 demonstrate that anti-paxillin $\mathrm{MAb}$ only recognizes the MBP-paxillin fusion and its derived proteolytic breakdown products present in bacterial extracts and does not recognize either GST-BE6 or other bacterial proteins. In lanes $4-7$ the indicated bacterial lysates were incubated together with glutathione agarose beads, and the washed beads were analysed for retained MBP-paxillin. GST-BE6 was able to associate with MBP-paxillin as well as with some of the MBPpaxillin degradation products, while GST alone showed no retention of MBP-paxillin (Figure 2 lane 4 compared to lane 6). This demonstrates that BE6 and 


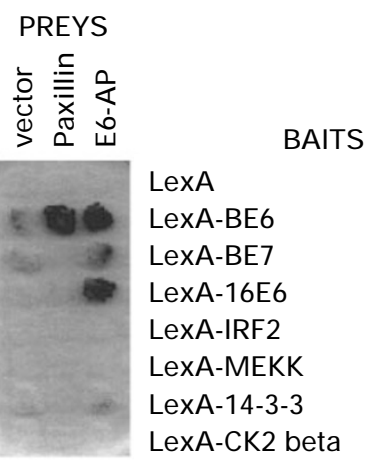

b

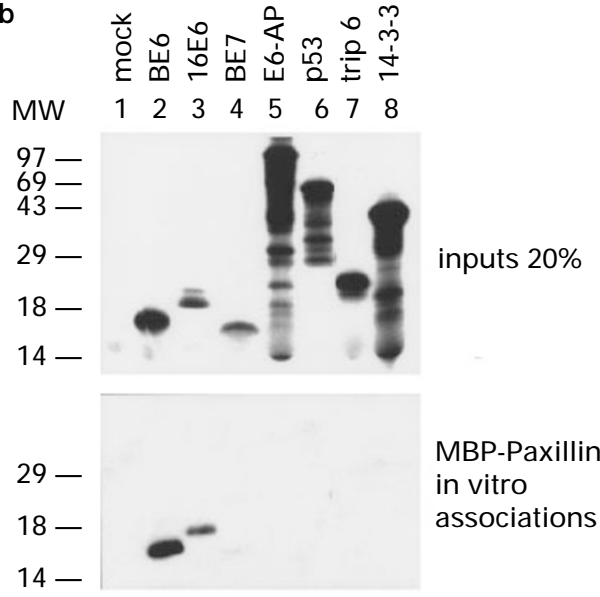

C

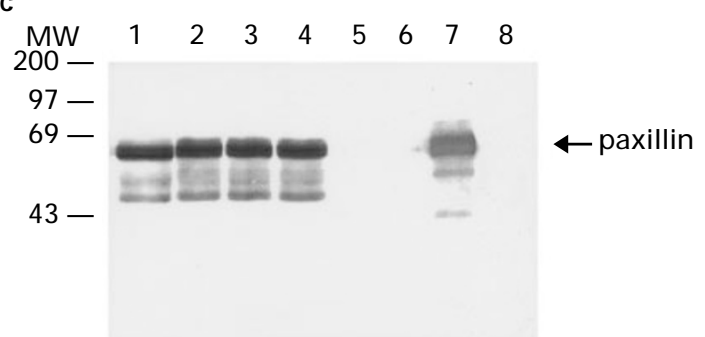

Figure 1 Paxillin association with BPV-1 E6 (BE6) in vivo and in vitro. (a) Association of paxillin and BE6 in yeast. The indicated lexA fusions (baits in (a) were cloned in frame with lexA in the yeast two hybrid plasmid pEG202 and introduced into the yeast reporter strain EGY48 together with a lexA responsive reporter plasmid pSH18-34 (Gyuris et al., 1993). BE6 refers to BPV-1 E6, BE7 to BPV-1 E7, 16E6 refers to the E6 oncogene of HPV-16, IRF2 refers to the transcriptional repressor IRF2 (Harada et al., 1994), MEKK1 is a human kinase (the generous gift of $\mathrm{Dr}$ Dennis Templeton), 14-3-3, (a signal transduction adapter protein) and ck2-beta (regulatory domain of casein kinase 2) are cDNAs isolated in our laboratory but are homologous to published human cDNAs (Heller-Harrison et al., 1989; Leffers et al., 1993; Vande Pol, unpublished observations). All of the indicated baits were shown to make lexA fusion proteins of the predicted size by Western blot analysis of yeast protein extracts using an antibody to lexA (not shown). The prey plasmids noted in the Figure express fusions of the indicated cDNAs to the B42 transcriptional activation domain in the yeast 2-hybrid prey plasmid pJG4-5 (Gyuris et al., 1993). EGY48 yeast strains expressing the indicated lexA fusions were mated to YPH499 yeast strains (Sikorski and Hieter, 1989) expressing the indicated prey plasmids. Diploids were isolated on selective media and replica-plated onto galactose plates containing XGAL as described (Gyuris et al., 1993). (b) In vitro association of paxillin and E6 oncogenes. In the upper panel, the indicated proteins were in vitro transcribed and translated in rabbit reticulocyte lsyates with $35 \mathrm{~S}$-cysteine, and $2 \mu \mathrm{l}$ of the translation reactions were analysed on 15\% SDS-PAGE gels followed by auto radiography. The lower panel shows the same in vitro translation reactions as the upper panel after in vitro association with MBP- paxillin are able to interact in the absence of other eukaryotic proteins, and indicates that BE6 can associate with paxillin that is not tyrosine phosphorylated.

\section{Paxillin interacts with a subset of E6 proteins}

In order to determine if the interaction of paxillin with E6 proteins is common among different papillomaviruses, the E6 proteins from BPV-1 (BE6), HPV16 and 18, and the rabbit cotton tail papillomavirus (CRPV) were tested in vitro for interaction with MBPpaxillin fusion protein. CRPV expresses two E6 proteins, a short (CRPVSE6) and long E6 (CRPVLE6) with translation of the shorter version initiated from an internal methionine (Meyers et al., 1992). Figure 3 demonstrates that only BE6 and 16E6 interacted with paxillin in this assay. Thus the interaction of BE6 with paxillin is neither universal nor a non-specific feature of papillomavirus E6 proteins.

\section{Paxillin interacts with BE6 through two separate interaction sites}

To identify domain(s) of paxillin that might interact with BE6, the full-length paxillin cDNA was cloned as an amino terminal fusion to the EE monoclonal antibody epitope in the plasmid pTM1 (Templeton, 1992). The resulting plasmid was digested with restriction enzymes and the restriction products in vitro transcribed and translated with 35-S methionine, generating c-terminally truncated paxillin proteins as shown in Figure 4a lanes 1-5. Lanes $1-5$ show the in vitro translation products for each of the restriction digests, which were tested for association with immobilized GST-BE6, as shown in lanes 6-10. The first 116 amino acids of paxillin retain the ability to bind GST-BE6 in vitro (Figure 4a lane 10). In order to more finely map the association of BE6 and paxillin, MBPpaxillin and GST-paxillin fusion proteins containing deletions of paxillin sequences were tested for association with wild-type BE6 (Figure 4b). While the first 79 amino acids of paxillin could interact with BE6, amino acids $12-79$ could not (Figure $4 b$ lanes 8 and 9), defining critical E6 interaction residues in the first 12 amino acids of paxillin. Surprisingly, Figure $4 \mathrm{~b}$ revealed the presence of at least one additional BE6 interaction

paxillin immobilized on agarose beads. The expressed cDNAs are the same as those described in (a) with the addition of the LIM domain containing protein trip6 (Lee et al., 1995), and wild-type human p53. (c) In vivo association of paxillin and BE6. GST-BE6 or GST-BE7 were transiently expressed in CV-1 cells by vaccinia/ pTM1 transfection as described (Elroy-Stein et al., 1989). Shown is a Western blot probed with monoclonal antibody 165 to paxillin. Samples in lanes $1-4$ are $20 \%$ of the solubilized total lysate in PBB buffer $(0.15 \mathrm{M} \mathrm{NaCl}, 50 \mathrm{~mm}$ Tris-Cl pH 7.4, $50 \mathrm{~mm}$ sodium fluoride, $5 \mathrm{~mm}$ sodium pyrophosphate, $0.5 \mathrm{~mm}$ sodium vanadate, $0.5 \mathrm{~mm}$ DTT, $0.5 \mathrm{~mm}$ PMSF, $1 \%$ NP40, $1 \mu \mathrm{g} / \mathrm{ml}$ aprotinin, $0.5 \mu \mathrm{g} / \mathrm{ml}$ leupeptin) from a $6 \mathrm{~cm}$ plate, while lanes $5-$ 8 are $80 \%$ of the same lysates after association of the lysate with glutathione agarose beads, washing of the beads in PBB and elution of the glutathione agarose beads with SDS-PAGE sample buffer. Lanes 1 and 5, mock infected; lanes 2 and 6 , infected and transfected with empty pTM1 plasmid; lanes 3 and 7 , infected and transfected with pTM1 plasmid expressing GSTBE6 fusion; lanes 4 and 8, infected and transfected with pTM1 plasmid expressing GST-BE7 fusion 


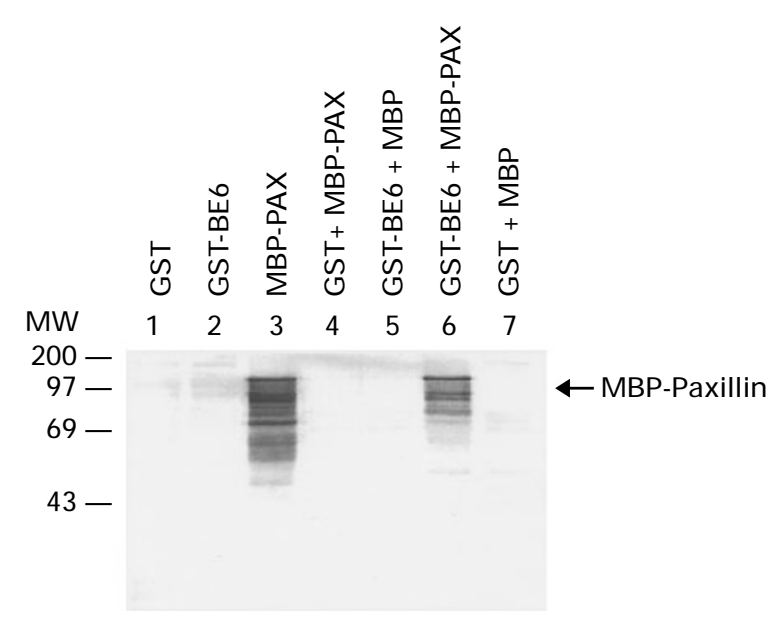

Figure 2 Association of BE6 and paxillin expressed in bacteria. The indicated glutathione-S-transferase fusions (GST) or maltose binding protein fusions (MBP) were expressed in E. coli, and crude bacterial lysates were prepared by sonication of bacteria in PBS $1 \%$ Triton X-100 with $0.5 \mathrm{~mm}$ PMSF followed by centrifugation of the extracts in a refrigerated microfuge for $10 \mathrm{~min}$. Lanes $1-3$ are bacterial lysate input samples. For lanes $4-7,5 \mu \mathrm{l}$ of the indicated bacterial extracts were incubated in $250 \mu \mathrm{l}$ of $\mathrm{PBB}$ at $4^{\circ} \mathrm{C}$ for $2 \mathrm{~h}$ followed by the addition of glutathione agarose beads for $30 \mathrm{~min}$. The beads were washed $4 \times$ with $1.0 \mathrm{ml}$ of PBB and the retained proteins solubilized in SDS sample buffer, resolved on $12 \%$ SDS-PAGE gels, transferred to PVDF membrane, and analysed by Western blot with monoclonal antibody 165 to paxillin

motif between amino acids 114-313 (Figure 4b lanes 5 and 7), distinct from the interaction motif found in amino acids $1-79$. Figure $4 \mathrm{~b}$ also reveals a low level of interaction of BE6 with the MBP-paxillin LIM domain region (lane 3), a region that mediates paxillin interactions with focal adhesions.

\section{E6-AP and paxillin are homologous in regions implicated in the binding of E6 proteins}

Previous studies of E6-AP had defined a 18 amino acid region necessary and sufficient for the interaction of E6-AP and E6 (Huibregtse et al., 1993b). This region was compared to paxillin amino acids $1-79$ and 114-323, with the results shown in Figure 4c. Two small regions in paxillin containing limited homology to E6-AP were in regions of paxillin implicated in association with BE6 in Figure 4a and b. The homologous regions in paxillin to E6-AP had been previously identified as sequence motifs termed LD motifs, for which a consensus sequence is shown in Figure 4c. There are $4 \mathrm{LD}$ motifs in paxillin; two (amino acids 144-156 and 266-278) were shown to mediate interaction with focal adhesion kinase (FAK) and one (amino acids 144-156) was also shown to interact with vinculin (Brown et al., 1996). The homology between E6-AP and paxillin is strongest for LD1 and LD2; however the data in Figure 4b does not discriminate among these motifs for interaction with BE6. LD1 sequences in amino acids $1-12$ are necessary for the binding of BE6 to GST-paxillin 179, (Figure 4b lanes 8 and 9), and in preliminary experiments not presented here, we have observed interaction of the first 15 amino acids of paxillin with immobilized MBP-BE6 in vitro.

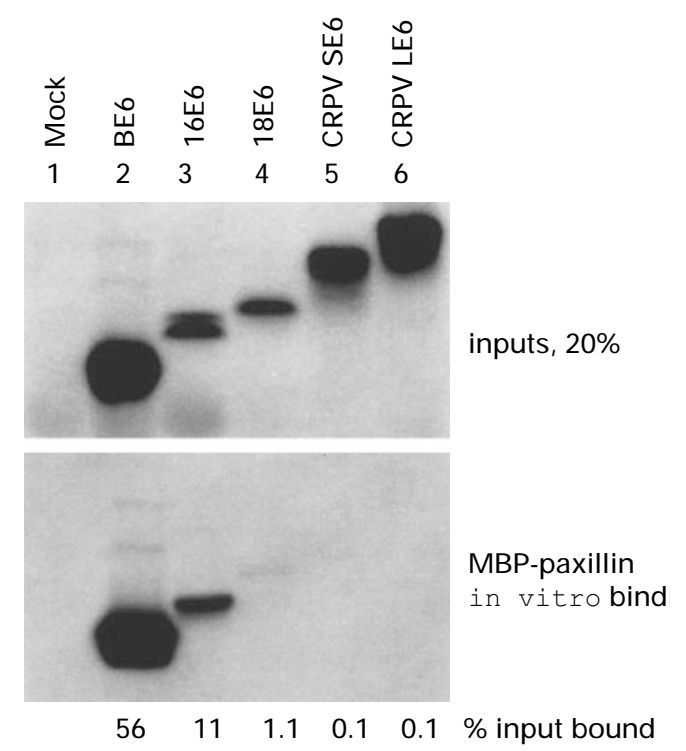

Figure 3 Paxillin association with E6 oncoproteins of differing papillomaviruses. The upper panel shows the products of in vitro transcription and translation reactions from rabbit reticulocyte lsyates programmed to express the indicated papillomavirus products. BE6 refers to BPV-1 E6, 16E6 to HPV-16 E6, 18E6 to HPV-18E8, CRPV SE6 to the short E6 protein from cotton tail rabbit papillomavirus (CRPV) and CRPV LE6 to the long E6 protein from CRPV (Meyers et al., 1992). The lower panel shows the same lysates after in vitro binding to and washing of MBPpaxillin-agarose beads. Protein content was quantified by beta counting of the dried gels. The numbers below the lower panel are the per cent of input counts immobilized on MBP-paxillin beads

\section{BE6 interaction with E6-AP and paxillin is mutually} exclusive

The similarity in the interaction motif in Figure $4 \mathrm{c}$ implies that E6-AP and paxillin may bind to the same region of BE6. In order to determine if BE6, E6-AP and paxillin can co-exist in a tri-molecular complex in vivo, GST-BE6 and ee-epitope-tagged paxillin and E6AP were overexpressed in CV-1 cells, and all three proteins were readily detected on Western blots (Figure $5 \mathrm{a}, \mathrm{b}$ and $\mathrm{c}$ ). Figure $5 \mathrm{~d}$ lanes 1 and 2 demonstrate the presence both ee-paxillin and ee-E6-AP bound to GSTBE6. When paxillin is immune-precipitated, BE6 is detected (Figure 5g), while ee-E6-AP is not detectable (Figure 5f), despite the presence of more ee-E6AP than ee-paxillin in the lysate (Figure 5c). These results indicate that GST-BE6 preferentially associated with paxillin compared to E6-AP, and that a tri-molecular complex of paxillin, E6-AP and BE6 was not detected in these assays.

\section{Interaction of BE6 with paxillin is associated with transformation}

If the interaction of paxillin and BE6 is essential for transformation, BE6 mutants that are greatly decreased for association with paxillin should be defective for transformation. A panel of BE6 point mutants were selected in yeast and characterized for transformation (Ned et al., 1997). These mutants were screened for association with E6-AP and paxillin in vivo by yeast two hybrid association and scored for induction of anchorage independent growth as shown in Figure 6. 
a

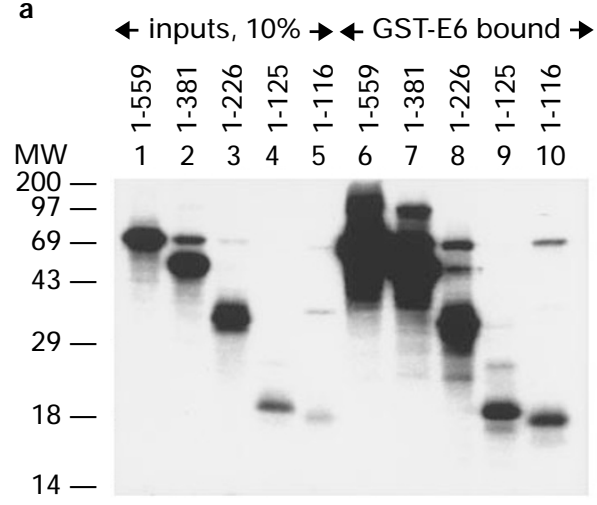

b

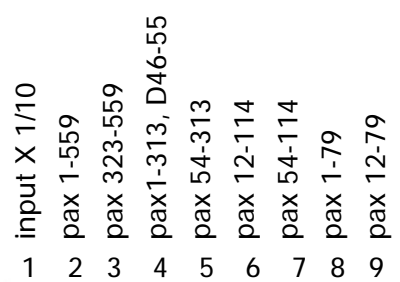

$\begin{array}{lllllllll}1 & 2 & 3 & 4 & 5 & 6 & 7 & 8 & 9\end{array}$

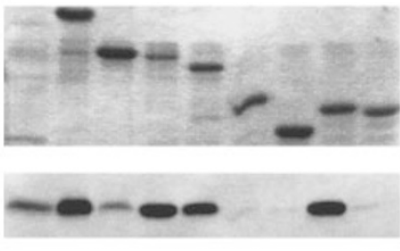

1001683493.03 .3824 .7

C

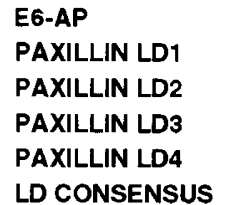

418

Figure 4 Mapping the BE6 binding domain of paxillin. (a) GSTBE6 association in vitro with C-terminally truncated paxillin. The full length chicken paxillin cDNA (559 amino acids) was cloned as an amino terminal fusion to the EE monoclonal antibody epitope in the pTM1 plasmid to yield pTM1-EE-paxilin. This plasmid wsa then digested with restriction enzymes and the resulting digested plasmids in vitro transcribed and translated with $35-\mathrm{S}$ methionine in rabbit reticulocyte lysate to generate run-off translation products, which were released from ribosomes with $0.1 \mu \mathrm{g}$ of puromycin. $10 \mu \mathrm{l}$ of the indicated programmed reticulocyte lysates were incubated in $250 \mu \mathrm{l}$ of PBB buffer with $1 \mu \mathrm{g}$ of GST-BE6 immobilized on glutathione agarose beads for $16 \mathrm{~h}$. The washed beads were re-suspended in SDS sample buffer and the products visualized by $15 \%$ SDS - PAGE and auto radiography. Lanes $1-5$ show $1 \mu \mathrm{l}$ of the input reticulocyte lysates. Lanes 2 and 7, pTM1EE-paxillin digested with EarI; lanes 3 and 8, digested with SacI; lanes 4 and 9, digested with Pst I; lanes 5 and 10, digested with BsrGI. (b) BE6 interaction with GST-paxillin deletion mutants. The indicated purified MBP-paxillin fusions (lanes 2 and 3) or GSTpaxillin fusions (lanes 4 -9) were immobilized on maltose agarose or glutathione agarose beads respectively and incubated in $250 \mu \mathrm{l}$ PBB for $16 \mathrm{~h}$ at $4{ }^{\circ} \mathrm{C}$ together with $10 \mu \mathrm{l}$ of rabbit reticulocyte lysate programmed to express 35-S labeled BE6. The beads were washed four times and re-suspended in SDS sample buffer with the eluted proteins resolved on a $15 \%$ SDS-PAGE. The upper panel shows the coumassie-stained gel, and the lower panel the auto radiogram of the same gel. Numbers below the auto radiogram show the per cent of input counts immobilized on the beads relative to MBPpaxillin 1-559 as determined by beta counting of the dried gel with a Packard Instant Imager. (c) Homology of paxillin to E6-AP in regions implicated in the binding of E6-AP to E6 oncoproteins. The 18 amino acids of E6-AP that associate with HPV-16 (Huibregtse $e t$ al., 1993b) are shown aligned to chicken paxillin sequences. The numbers refer to the last amino acid in the shown segments

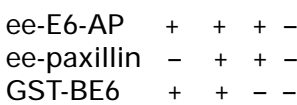

$1 \quad 2334$

Precipitation Western Blot

A

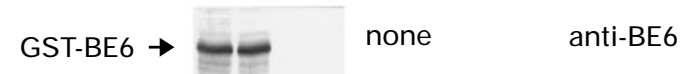

B

Paxillin $\rightarrow$ none paxillin MAb

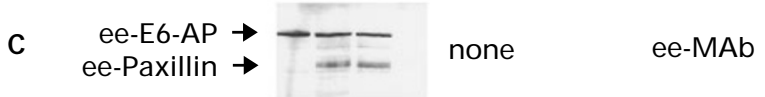

D ee-E6-AP $\rightarrow--\quad$ GT-agarose ee-MAb

E ee-Paxillin $\rightarrow$ ee-MAb paxillin MAb

F ee-Paxillin $\rightarrow \quad$ paxillin MAb ee-MAb

G $\begin{aligned} \lg G & \rightarrow \\ \lg G & \rightarrow \\ \text { GST-BE6 } & \rightarrow\end{aligned}$ paxillin MAb anti-BE6

H

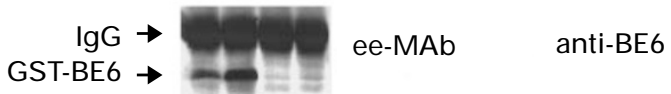

Figure 5 Binding of E6-AP and paxillin to BE6 is mutually exclusive. The indicated plasmids expressing GST-BE6, and E6$\mathrm{AP}$ and paxillin tagged at the amino terminus with the ee MAb epitope (ee-paxillin and ee-E6-AP respectively) were co-expressed in monkey kidney CV-1 cells by vaccinia-pTM1 transfection (Elroy-Stein et al., 1989). Cells were lysed in $0.5 \times \mathrm{PBB}$ and clarified lysates either analysed directly by Western blot with the indicated antibodies (parts $\mathbf{A}, \mathbf{B}$ and $\mathbf{C}$ ) or subjected to immune precipitation with the indicated antibodies together with bridging rabbit anti-mouse and protein A sepharose (parts E, F, G and $\mathbf{H}$ ). Monoclonal antibodies to paxillin (Transduction Laboratories), ee epitope (Walter et al., 1990), and rabbit polyclonal antibody to BE6 (Ned et al., 1997) were used for precipitation and detection. GST-BE6 and associated proteins were precipitated with glutathione-agarose beads (part D). Precipitates were analysed by SDS-PAGE followed by blotting to PVDF membranes and detection with the indicated antibodies. The migration of GSTBE6, ee-paxillin, ee-E6-AP and IgG heavy chain are indicated by arrowheads left of the blots

All BE6 mutants that were greatly diminished for interaction with either E6-AP or paxillin in yeast were defective for the ability to induce anchorage independence in the contact-inhibited $\mathrm{C} 127$ cell line. All of the BE6 mutants that retained interaction with E6-AP also retained association with paxillin in yeast. However, several mutants showed significant interaction with paxillin while not interacting with E6-AP (F37S, $\Delta 135-7$, P137R, L83P), demonstrating that mutant BE6 proteins can discriminate between E6-AP and paxillin. Figure 6 reveals that only BE6 mutants R42W，K114E, R129S and $\Delta 131-133$ could induce anchorage independence at near wild-type levels, and all of these mutants retained some association with both E6-AP and paxillin. Mutants showing association with paxillin in vivo while losing association with E6AP (F37S, $\Delta 135-7, \quad$ P137R and L83P) were all defective for anchorage independence. Interaction with both E6-AP and paxillin in vivo was necessary but not sufficient for inducing anchorage independence as BE6 mutants K35E, and $\mathrm{R} 123 \mathrm{H}$ were defective for 


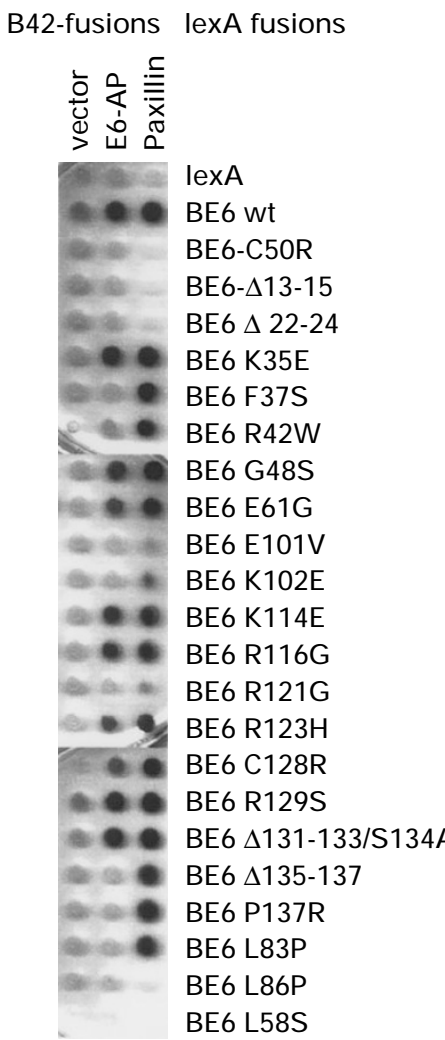

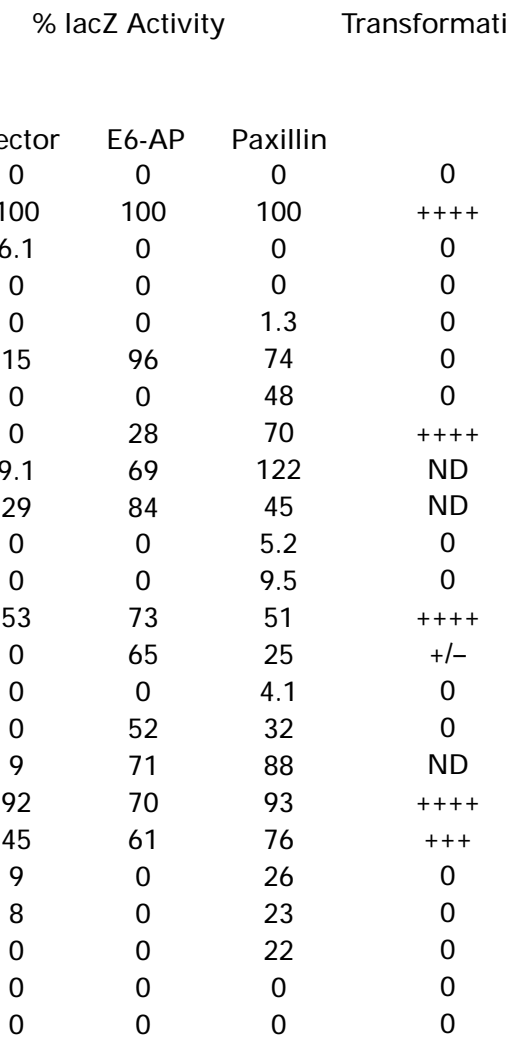

Figure 6 In vivo interaction of paxillin with BE6 and BE6 mutants, and correlation with transformation. BE6 and BE6 mutants cloned as lexA fusions were introduced into the yeast reporter strain EGY48 together with the lexA-responsive lacZ reporter plasmid pSH18-34 (Gyuris et al., 1993). The resulting yeast strains were separately mated to YPH499 yeast strains containing plasmids that express B42-transactivation domain fusions to either E6-AP, paxillin, or the empty plasmid (vector). The resulting diploids were isolated on selective plates and individually grown in liquid glucose selective media for $4 \mathrm{~h}$ prior to spotting of the yeast cultures onto XGAL-containing galactose plates (the B42 fusion plasmid is induced by galactose), for a graphic indication of the 2-hybrid associations. Quantitative lacZ assays were as described in the Materials and methods and in (Kippert, 1995). The results shown are the lacZ activity relative to that obtained with wild-type lexA-BE6 fusion. Because BE6-lexA fusions have endogenous transcriptional activation properties which are altered in BE6 mutants (Lamberti et al., 1990), OD405 values obtained in cultures expressing the empty B42-fusion vector alone were substracted from values obtained in the presence of B42-E6-AP or B42-paxillin fusions, to more closely approximate the lacZ activity resulting from the interaction of lexA-BE6 with E6-AP or paxillin. Transformation results reflect the induction of anchorage independent growth in nobel agar by BE6 mutants relative to wild-type BE6. Equal numbers of pooled G418-resistant mouse C127 cells 14 days after transfection with either BE6 or BE6 mutants expressed from the retroviral expression plasmid pLXSN were seeded into agar as previously described (Vande Pol and Howley, 1995). Cultures were scored for anchorage independence 10-14 days later. Results shown are the product of at least three separate transfections for each mutant and are expressed as anchorage independence relative to wild-type BE6. ++++ refers to colonies of equivalent size and at least $50 \%$ of the frequency of wild-type BE6; +++ refers to colonies of at least $20 \%$ the frequency and similar size as wild-type BE6; ++ refers to colonies distinctly smaller than wild-type BE6 that arise at less than $10 \%$ the frequency of wild-type BE6; + / - refers to small colonies that arise at $1 \%$ or less than the frequency of wild-type; 0 refers to no colonies above that seen with the empty vector pLXSN. Pooled G418-resistant colonies transfected with wild-type BE6 induced anchorage independent colonies at 55-70\% efficiency in these assays. The results shown are the results from Ned et al. (1997)

anchorage independence yet retained association with both E6-AP and paxillin.

\section{BE6 transformed cells are altered in paxillin phosphorylation}

In cells transformed by v-src or bcr-abl, tyrosine phosphorylation of paxillin is dramatically increased, contributing to speculation that paxillin may mediate some of these oncogene's effects. In order to determine if paxillin levels or phosphorylation are altered in BE6 transformed cells, mouse C127 cells were transfected wild-type BE6, mutant BE6 $\Delta 13-15$ (transformation defective and non-binding to paxillin) or vector alone. No difference was noted in paxillin content or phosphotyrosine levels as determined by Western blot (Figure 7a). In order to determine if changes in phosphorylation of paxillin were associated with BE6 transformation of $\mathrm{C} 127$ cells, the same cell lines used in Figure $7 \mathrm{a}$ were metabolically labeled with ${ }^{32} \mathrm{P}$-orthophosphate, paxillin was immune-precipitated and subjected to phospho-amino acid analysis as shown in Figure 7b. A twofold increase in phosphoserine and corresponding decrease in phosphothreonine content was observed in cells transfected with wild-type BE6 compared to the non-transforming BE6 mutant BE6 $\Delta 13-15$, but there was little difference in phosphotyrosine content.

\section{Discussion}

In this study we have found an association between the E6 oncoprotein and paxillin, a protein implicated in integrin signaling. Transformation of contact inhibited 

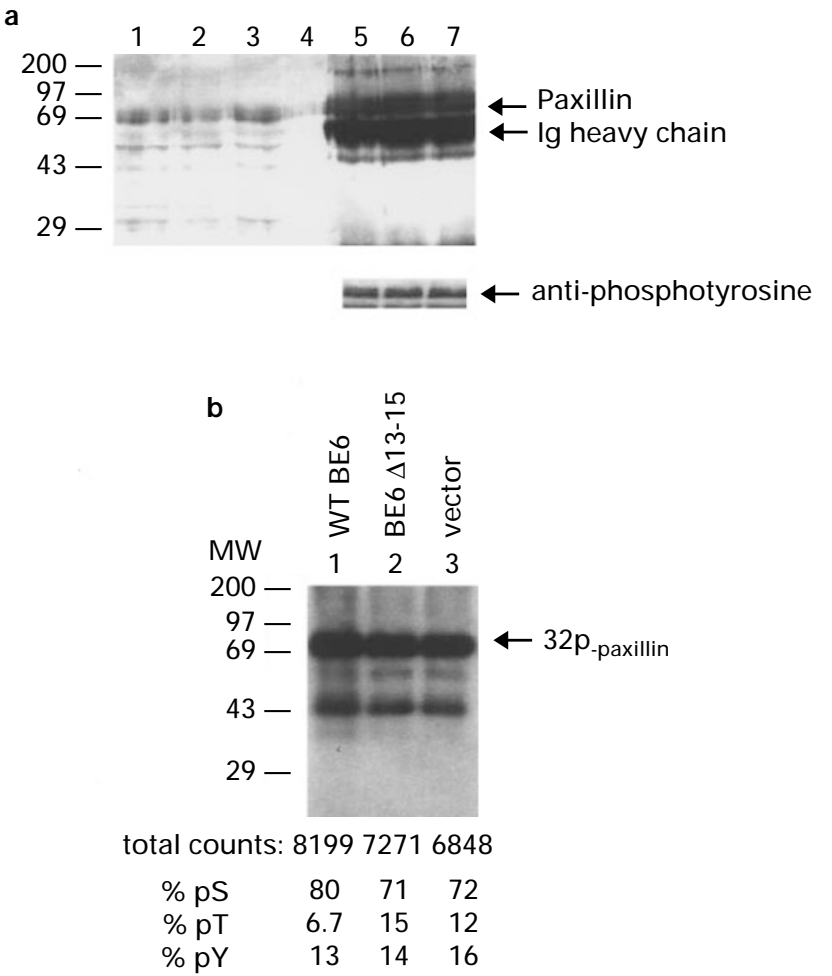

Figure 7 (a) Levels of paxillin in BE6 transformed cells. Equal numbers of pooled $\mathrm{G} 418$ resistant $\mathrm{C} 127$ cells transfected with the retroviral expression in plasmid pLXSN expressing wild-type BE6 (pLXSN-BE6, lanes 1 and 5), a transformation defective BE6 mutant (pLXSN-BE6 $\Delta 13-15$, lanes 3 and 7 ) or the empty plasmid pLXSN (lanes 2 and 6) were lysed in PBB. The clarified lysates were analysed directly (lanes $1-3$ ) or after immune precipitation with antibody to paxillin (anti-paxillin MAb, Transduction Laboratories) by SDS-PAGE followed by transfer to PVDF membranes and Western blot analysis with MAb to paxillin. In the lower panel, the same lysate samples first immune precipitated with anti-paxillin antibody were analysed for phosphotyrosine content utilizing a monoclonal anti-phosphotyrosine antibody by Western blot (MAb PY20, Transduction Laboratories). (b) Phosphorylation of paxillin in C127 cells and BE6 transformed C127 cells. Equal numbers of pooled G418 resistant C127 cells transfected with the retroviral expression plasmid pLXSN (lane 3), expressing wild-type BE6 (pLXSN-BE6, lane 1), or a transformation defective BE6 mutant (pLXSN-BE6 $\Delta 13-15$, lane 2) or the empty plasmid pLXSN (lane 3) were metabolically labeled with ${ }^{32} \mathrm{P}$ orthophosphate at $1.0 \mathrm{mCi} / \mathrm{ml}$ for $4 \mathrm{~h}$ prior to lysis in PBB. The lysates were pre-cleared with protein A/G sepharose and a non-specific mouse monoclonal antibody (antihemagglutanin MAb 12CA5) prior to immune precipitation of paxillin with mouse monoclonal antibody to paxillin. The immune precipitates were resolved on $10 \%$ SDS-PAGE gels and transferred to PVDF membranes which were analysed by auto radiography and scanned by a Packard Instant Imager to quantitate ${ }^{32} \mathrm{P}$. The paxillin bands were excised and phosphoamino acids determined as described (Kamps and Sefton, 1989) and quantified by beta-scanning of electrophoresis plates. The relative phospho-amino acid content for each sample is indicated below the corresponding lane

cells by oncogenes is accompanied by changes in the cytoskeleton. Paxillin association with focal adhesion proteins (FAK and vinculin), its localization in focal adhesions, and its presence as a substrate of provocative oncogenes and proto-oncogenes (v-src, csrc, v-crk, crkl, bcr-abl) makes it plausible that paxillin exerts a regulatory role in the formation of focal adhesions, the remodeling of the cytoskeleton and transformation. We identified paxillin as an interactor with BE6 in a 2-hybrid screen in yeast. In vitro association of paxillin and BE6 expressed in bacteria confirmed that the BE6-paxillin association is independent of the presence of other eukaryotic proteins (Figure 2). Like E6-AP, the association of paxillin with E6 proteins is restricted to a subset of papillomavirus types (Figure 3 and reference (Huibregtse et al., 1993a)). This is another example of the surprising biochemical diversity exhibited by papillomavirus E6 proteins. Associations of E6-AP, ERC-55 and now paxillin have all demonstrated restricted interactions to only a subset of papillomavirus E6 proteins (Figure 3, and references (Chen et al., 1995; Huibregtse et al., 1993a)). While the role of paxillin and ERC-55 associations with E6 in the papillomavirus life cycle is as yet undetermined, E6-AP association is clearly implicated in the life cycle of papillomaviruses through the targeted degradation of p53. Yet E6-AP associates with E6 proteins from only a subset of papillomaviruses indicating that the biological importance of an association does not require that the association be universal among E6 proteins. This restriction of interactions to only a subset of E6 proteins is unlike papillomavirus E7 proteins as well as the TAg and E1a oncoproteins of papovaviruses and adenovirus respectively that interact with a common set of cellular proteins (Howley et al., 1991).

There are several surprising features of the BE6paxillin interaction. Homologous sequences between paxillin and E6-AP were implicated in the interaction of paxillin with BE6. These sequences in paxillin, previously called LD motifs, were first described as sequences in paxillin that bound to focal adhesion kinase and vinculin (Brown et al., 1996). The homology between paxillin and E6-AP reveals that there is a protein interaction sequence common to E6AP and paxillin that does not share all of the features of the LD motifs (Figure 4c). The further characterization of this sequence element may aid in the identification of additional E6 binding proteins, and determine if this motif is a general protein interaction motif or if this motif is common to proteins with shared functionality (such as the LXCXE motif found in proteins that interact with the retinoblastoma family of proteins (Harlow, 1990).

How BE6 might interact with the LD-like motif in E6-AP and the LD motif in paxillin is unknown, but the mutational analysis performed here (Figure 6) indicates that sequences throughout the BE6 primary amino acid sequence contribute to interaction with both E6-AP and paxillin, implying that BE6 may adopt a complex folded shape to interact with paxillin, and not interact through a short peptide motif in BE6. The fact that BE6 can interact with more than one site on paxillin (Figure 4) makes the interpretation of the BE6paxillin complex potentially complex: BE6 binding to paxillin may have different consequences depending upon the site bound, the BE6 binding sites on paxillin may vary if their affinity for BE6, and BE6 mutants may be differentially altered in their association with different sites on paxillin.

What might be the consequences of the BE6-paxillin association? In the case of E6-AP and p53, E6 acts as an adaptor protein bringing p53 into contact with the E6-AP ubiquitin ligase. Based upon this model, one might predict that BE6 is bringing a modifying function such as a kinase to paxillin or perhaps using 
paxillin to gain access to an as yet identified protein. The alteration in paxillin phosphoserine content in BE6 transformed cells is suggestive of this possibility. Serine phosphorylation of paxillin has recently been described as a result of integrin signaling, indicating that phosphorylation of paxillin associated with the formation of focal adhesions can in some circumstances involve serine and not tyrosine residues (Bellis et al., 1997; De Nichilo and Yamada, 1996). The change in paxillin phosphoserine content in BE6 transformed cells was significant but not dramatic, indicating that potential BE6 regulation of paxillin function through altered phosphorylation may be subtle, and/or restricted to particular sites of paxillin. We did not observe dramatic differences in focal adhesions or stress fibers in BE6 transformed $\mathrm{C} 127$ cells compared to untransformed $\mathrm{C} 127$ cells (unpublished observations). BE6 may compete with other cell regulatory proteins for binding to paxillin since the LD motifs of paxillin have recently been shown to mediate the interaction of paxillin with FAK and vinculin (Brown et al., 1996). The BE6-binding LD1 at the extreme amino terminus of paxillin (amino acids $1-15$ ) does not yet have an ascribed function, but it is close to tyrosine residues phosphorylated by FAK (amino acids 31 and 118) and c-src in vitro that create SH2binding sites for the Crk adaptor protein (Bellis et al., 1997; Schaller and Parsons, 1995).

The evidence that the association of BE6 and paxillin may play a role in induction of anchorage independence by BE6 is biologically plausible, given the association of paxillin with focal adhesions and the postulated role of paxillin in integrin signaling events. BE6 mutants that induce anchorage independence all associate with paxillin, and BE6 mutants that failed to associate with paxillin do not induce anchorage independence (Figure 6). The interaction of E6-AP and paxillin with BE6 is mutually exclusive in vitro (Figure 7), raising the possibility that the interaction of BE6 with paxillin may repress the interaction of BE6 with E6-AP, or vice versa. Alternatively, the interaction of BE6 with paxillin and E6-AP may be separate and complementing effects in the induction of anchorage independence by BE6.

Recently, another group has independently demonstrated the association of BE6 and HPV-16 E6 with paxillin after immune precipitation of BE6 and microsequencing of associated proteins (Tong and Howley, 1997).

\section{Materials and methods}

\section{Plasmids}

Paxillin clones: The full-length chicken paxillin cDNA clone (paxillin 11-559) (Turner and Miller, 1994) were pcr amplified and cloned into the EcoRI and XhoI sites of pJG4-5 to create a paxillin fusion to the B42 transactivation domain for expression in a yeast two hybrid system (Gyuris et al., 1993). Maltose binding protein (MBP) paxillin fusion for expression in $E$. coli was created by per amplification of the full length paxillin cDNA and was cloned as a $E c o$ RI to $B a m \mathrm{HI}$ fragment into pMAL-cR1 (New England Biolabs). MBP-paxillin fusion amino acids 3231-559 was per amplified and cloned as a EcoRI to BamHI fragment into pMAL-cR1. GST-paxillin fusions have been previously described (Brown et al., 1996). The full-length paxillin cDNA from pJG-paxillin was cloned as a EcoRI to XhoI fragment into a modified pTM1-5' ee plasmid (Elroy-Stein et al., 1989; Yan and Templeton, 1994), where paxillin is fused to the ee monoclonal antibody epitope (Walter et al., 1990). GST fusions to BE6 and to BPV-1 E7 (BE7) were cloned into pTM1 for expression in mammalian cells by T7-vaccinia virus expression (Elroy-Stein et al., 1989), and into pGEX-4T1 (Pharmacia) for expression in E. coli. BE6 point and deletion mutants are from (Ned et al., 1997). For transformation assays, BE6 and BE6 mutants were expressed from the retroviral expression plasmid pLXSN (Miller and Rosman, 1989). BE6, BE7, and the E6 genes from HPV-16, 18, 11, and cotton tail rabbit papillomavirus (CRPV) were pcr amplified with primers containing $5^{\prime}$ EcoRI and $3^{\prime} X$ Xho I sites and cloned into pTM1 for in vitro transcription and translation, and into pEG202 for expression as lexA fusions in yeast (Gyuris et al., 1993). The cDNA for E6-AP was the generous gift of Dr John Huibregtse (Rutgers University), and was cloned into pTM1-5' ee for in vitro transcription and translation. A yeast 2 micron expression plasmid expressing lexA fusions from the ADH promoter with URA3 selection was created by pcr amplification of the $1301 \mathrm{nt} S p h \mathrm{I}$ fragment containing the $\mathrm{ADH}$ promoter, lexA open reading frame and ADH 1 terminator with $5^{\prime} \mathrm{SacI}$ and $3^{\prime} \mathrm{KpnI}$ cloning sites from the plasmid pEG202; the resulting pcr fragment was cloned into the $S a c I$ and $K p n I$ sites of the yeast 2 micron URA3 plasmid pRS426 (Sikorski and Hieter, 1989), to create p1704A.

\section{Cell lines, transfections and culture conditions}

Monkey kidney CV-1 cells and mouse C127 cell lines were maintained in DMEM media supplemented with $10 \%$ newborn calf serum, glutamine and antibiotics. Vaccinia virus pTM1 transfections were performed as described (Rose et al., 1991).

\section{Yeast expression systems}

BE6 and BE6 mutants were cloned as lexA fusions into the lexA DNA binding domain yeast expression plasmid pEG202 (Gyuris et al., 1993). pEG202 and the lexA responsive LacZ reporter plasmid pSH18-34 were the generous gift of Roger Brent (Harvard University). Human E6-AP (provided by John Huibregtse, Rutgers University) was cloned as a fusion to the B42 transactivation domain in the yeast 2-hybrid prey plasmid pJG4-5 (Gyuris et al., 1993). All yeast media, culture conditions and transfections were done with standard techniques (Ausubel et al., 1995).

\section{2-hybrid assay}

The L40 strain of yeast (Hollenberg et al., 1995) expressing a lexA-VP16 fusion was EMS mutagenized (Lawrence, 1991) and a blue colony ura 3 mutant was isolated on a selective plate containing uracil, FOA (Boeke et al., 1987) and XGAL. The resulting strain of yeast was cured of the lexA-VP16 plasmid by growth in the presence of tryptophan, and a white colony was selected on a plate containing tryptophan, leucine, adenine, histidine, uracil, FOA and XGAL, resulting in the strain L40A5. L40A5 has the same genotype as L40 but is ura3 from the EMS mutagenesis. A yeast cDNA expression library was created in the plasmid pJG 4-5 as follows: $5 \mu \mathrm{g}$ of polyA-selected mRNA from a HPV-18 immortalized human keratinocyte cell line (Romanczuk et al., 1991) was reverse transcribed utilizing both a random primer with a $3^{\prime}$ Xho cloning site (GAGAGAGAGAGACTCGAGNNNNNNNN) and an 
oligo dT primer with a $3^{\prime}$ Xho cloning site, converted to double stranded cDNA with EcoRI adapters utilizing a commercial cDNA synthesis kit (Gibco-BRL), 5-methyldCTP, and ligated into EcoRI and XhoI cut pJG4-5 according to the manufacturer's directions and (Gubler and Hoffman, 1983). $1.4 \times 10^{6}$ primary colonies resulted from electroporation of the ligated cDNA. A lex-BE6 fusion in the yeast 2 micron URA3 plasmid p1704A was created by cloning BE6 in frame to lexA in the EcoRI and XhoI sites of p1704A, resulting in the plasmid p1704ABE6. This was introduced into yeast strain L40A5. The human keratinocyte cDNA library in pJG4-5 was transfected into the resulting strain and colonies selected on CM plates minus histidine, uracil, tryptophan, and containing $1 \%$ galactose and $2 \%$ raffinose as the carbon source, and $50 \mathrm{~mm} \mathrm{3-aminotriazole.} \mathrm{Colonies} \mathrm{were} \mathrm{lifted} \mathrm{on}$ nitrocellulose filters from galactose-raffinose CM plates minus histidine and transferred to galactose plates containing XGAL. Blue colonies were picked and transferred to glucose CM plates containing histidine, uracil, FOA, and leucine to select for loss of the lexA-BE6 bait plasmid. The resulting colonies were mated to $\mathrm{YPH} 500$ yeast containing a lexA-BE7 on a URA3 plasmid, diploids were selected and screened on galactose plates containing XGAL. White colonies were selected, containing potential prey cDNA expressing plasmids that interacted with lexA-BE6 but not lexA-BE7.

\section{Yeast beta-galactosidase assays}

These were performed in 96 well microtiter plates as follows: Yeast were inoculated into $0.1 \mathrm{ml}$ of selective media with raffinose as the carbon source diluted $1: 10$ with sterile water and grown to saturation overnight. (Raffinose is used instead of glucose to avoid repression of the prey plasmid Gall promoter by glucose). In the morning $0.05 \mathrm{ml}$ of $3 \times$ selective media with raffinose as a carbon source was added to each well and the plates grown at $30{ }^{\circ} \mathrm{C}$ for $3 \mathrm{~h} .0 .02 \mathrm{ml}$ of $20 \%$ galactose was added per well to induce the galactose-inducible promoter on the prey plasmids and the cells were incubated at $30^{\circ} \mathrm{C}$ for another $6 \mathrm{~h}$. The plates were then centrifuged, the media discarded, and the yeast re suspended with $0.2 \mathrm{ml}$ of sterile water. The plates were then read with a 96 well spectrophotometer at $650 \mathrm{nM}$ to determine the yeast concentration in each well, and the plates subsequently re-centrifuged and the water discarded. The yeast were then re-suspended in $0.1 \mathrm{ml}$ per well of beta galactosidase assay buffer ( $\mathrm{Z}$ buffer) containing ONPG and $0.2 \%$ sarkosyl to permeabilize the yeast (Kippert, 1995). After the development of yellow color, the reaction was stopped with $0.1 \mathrm{ml}$ of $0.1 \mathrm{M}$ sodium carbonate, the plates were re-centrifuged, and $0.1 \mathrm{ml}$ per well removed to a new microtiter plate and the plate optical density read at $410 \mathrm{nM}$.

\section{References}

Ausubel F, Brent R, Kingston R, Moore D, Seidman J, Smith J and Struhl K. (1995). Current Protocols in Molecular Biology. John Wiley and Sons, Inc.

Bellis SL, Miller JT and Turner CE. (1995). J. Biol. Chem., 270, $17437-17441$.

Bellis SL, Perrotta JA, Curtis MS and Turner CE. (1997). Biochem. J. 325: $375-381$.

Birge RB, Fajardo JE, Reichman C, Shoelson SE, Songyang Z, Cantley LC and Hanafusa H. (1993). Mol. Cell. Biol., 13, $4648-4656$.

Boeke JD, Trueheart J, Natsoulis G and Fink GR. (1987). Methods Enzymol, 154, 164-175.
Immune precipitations and Western blots

Cells were lysed in $1.0 \mathrm{ml}$ of PBB buffer $(0.15 \mathrm{M} \mathrm{NaCl}$, $0.05 \mathrm{M}$ Tris $\mathrm{Cl} \mathrm{pH} \mathrm{7.5,} 50 \mathrm{~mm} \mathrm{NaF}, 5 \mathrm{~mm} \mathrm{Na}$ Pyrophosphate, $0.5 \mathrm{~mm}$ sodium vanadate, $0.5 \mathrm{mM}$ DTT, $0.5 \mathrm{~mm}$ PMSF, $1 \%$ NP40, $1 \mu \mathrm{g} / \mathrm{ml}$ aprotinin, $0.5 \mu \mathrm{g} / \mathrm{ml}$ leupeptin) per $10^{7}$ cells on ice for $30 \mathrm{~min}$ and the lysates clarified by centrifugation in a microfuge at $4^{\circ} \mathrm{C}$ for $10 \mathrm{~min}$. Immune precipitation with monoclonal antibodies to paxillin (MAb 165 (Turner et al., 1990) and MAb to paxillin from Transduction Laboratories, Inc.) was performed with $0.5 \mu \mathrm{g}$ of monoclonal antibody, bridging rabbit antimouse antibody and protein A sepharose beads. SDSPAGE gels were transferred to PVDF membranes, probed with monoclonal antibodies to paxillin or phosphotyrosine (a mix of PY20, Transduction Laboratories, Inc., and Ab-2 from Oncogene Science); detection of bound antibody was with goat anti-mouse coupled to alkaline phosphatase and $\mathrm{BCIP} / \mathrm{NBT}$ colorimetric detection.

\section{In vitro translation and in vitro binding assays}

In vitro coupled transcriptions and translations were performed utilizing standard nuclease treated reticulocyte lysate (Promega) according to the manufacturer's recommendations, supplemented with $1.5 \mathrm{mM} \mathrm{MgCl}_{2} 2.5 \mathrm{~mm}$ nucleotide triphosphates and 25 units T7 RNA polymerase (Gibco-BRL) per $50 \mu \mathrm{l}$ translation reaction. For in vitro binding assays approximately $1-5 \mu \mathrm{g}$ of MPB or GST fusions immobilized on agarose beads were suspended in $150 \mu \mathrm{l} \mathrm{PBB}$ buffer (for MBP-paxillin fusions) or $0.5 \times \mathrm{PBB}$ buffer (for GST-E6-AP fusions) and $10 \mu \mathrm{l}$ reticulocyte lysate programmed to express the indicated proteins was added. The samples were incubated for $16 \mathrm{~h}$ at $4^{\circ} \mathrm{C}$, and the beads washed three times with $1.0 \mathrm{ml}$ of the appropriate binding buffer. Retained proteins were eluted with SDS sample buffer, resolved by $15 \%$ SDS-PAGE, fluorographed with salicylate (Chamberlain, 1979), and subjected to autoradiography. Where indicated, radioactive proteins were quantitated by gel scanning with a Packard Instant Imager.

\section{Acknowledgements}

The authors thank Stanley Hollenberg for the L40 yeast strain, Dr John Huibregtse for the E6-AP cDNA, Joseph Parrotta for paxillin constructs and mutants, and Dr Dennis Templeton for guidance on phospho-amino acid analysis, vaccinia virus pTM1 expression, and helpful discussions. The authors thank Drs Adam Goldfarb, Dennis Templeton, and David Kaplan for critical reading of the manuscript. This work was supported by grants NIH CA69292 to SV and NIH GM47607 to CET. CET is an established investigator of the American Heart Association. $\mathrm{MCB}$ is an AHA postdoctoral fellow.

Brown MC, Perotta JA and Turner CE. (1996). J. Cell Biol., 135, $1109-1123$.

Chamberlain JP. (1979). Anal. Biochem., 98, $132-135$.

Chen JJ, Reid CE, Band V and Androphy EJ. (1995). Science, 269, 529-531.

Dalal S, Gao Q, Androphy EJ and Band V. (1996). J. Virol., 70, $683-688$

De Nichilo MO and Yamada KM. (1996). J. Biol. Chem., 271, 11016-11022.

Elroy-Stein O, Fuerst TR and Moss B. (1989). Proc. Natl. Acad. Sci. USA, 86, 6126-6130. 
Glenney JR and Zokas L. (1989). J. Cell. Biol., 108, $2401-$ 2408.

Gubler U and Hoffman BJ. (1983). Gene, 25, 263-269.

Gyuris J, Golemis E, Chertkov H and Brent R. (1993). Cell, 75, $791-803$.

Harada H, Takahashi E, Itoh S, Harada K, Hori TA and Taniguchi T. (1994). Mol. Cell. Biol., 14, 1500-1509.

Harlow E. (1990). Ciba Found. Symp., 150, $262-271$.

Heller-Harrison RA, Meisner H and Czech MP. (1989). Biochemistry, 28, 9053-9058.

Hildebrand JD, Schaller MD and Parsons JT. (1995). Mol. Biol. Cell., 6, 637-647.

Hollenberg SM, Sternglanz R, Cheng PF and Weintraub H. (1995). Mol. Cell. Biol., 15, 3813-3822.

Howley PM, Munger K, Romanczuk H, Scheffner M and Huibregtse JM. (1991). Princess Takamatsu Symp., 22, $239-248$

Huibregtse JM, Scheffner M and Howley PM. (1991). EMBO $J ., \mathbf{1 0}, 4129-4135$.

Huibregtse JM, Scheffner M and Howley PM. (1993a). Mol. Cell. Biol., 13, 775-784.

Huibregtse JM, Scheffner M and Howley PM. (1993b). Mol. Cell. Biol., 13, 4918-4927.

Kamps MP and Sefton BM. (1989). Anal. Biochem., 176, $22-$ 27.

Kippert F. (1995). FEMS Microbiol. Lett., 128, $201-206$.

Lamberti C, Morrissey LC, Grossman SR and Androphy EJ. (1990). ЕMBO J., 9, 1907-1913.

Lawrence C. (1991). Methods Enzym., 194, 273-281.

Lee JW, Choi HS, Gyuris J, Brent R and Moore DD. (1995). Mol. Endocrinol., 9, 243-254.

Leffers H, Madsen P, Rasmussen HH, Honore B, Andersen AH, Walbum E, Vandekerckhove J and Celis JE. (1993). J. Mol. Biol., 231, $982-998$.

Meyers C, Harry J, Lin YL and Wettstein FO. (1992). $J$. Virol., 66, $1655-1664$.

Miller AD and Rosman GJ. (1989). BioTechniques, 7, 980 990.

Ned R, Allen S and Vande Pol S. (1997). J. Virol., 71, $4866-$ 4870.

Romanczuk H, Villa LL, Schlegel R and Howley PM. (1991). J. Virol., 65, 2739- 2744 .
Rose JK, Buonocore L and Whitt MA. (1991). BioTechniques, 10, $520-525$.

Sabe H, Hata A, Okada M, Nakagawa $\mathrm{H}$ and Hanafusa $\mathrm{H}$. (1994). Proc. Natl. Acad. Sci. USA, 91, 3984-3988.

Salgia R, Li JL, Lo SH, Brunkhorst B, Kansas GS, Sobhany ES, Sun Y, Pisick E, Hallek M, Ernst T et al. (1995a). J. Biol. Chem., 270, $5039-5047$.

Salgia R, Uemura N, Okuda K, Li JL, Pisick E, Sattler M, de Jong R, Druker B, Heisterkamp N, Chen LB et al. (1995b). J. Biol. Chem., 270, 29145-29150.

Schaller MD and Parsons JT. (1995). Mol. Cell. Biol., 15, $2635-2645$

Scheffner M, Munger K, Huibregtse J and Howley P. (1992). EMBO J., 11, 2425-2431.

Schiller JT, Vass WC and Lowy DR. (1984). Proc. Natl. Acad. Sci. USA, 81, 7880-7884.

Sikorski RS and Hieter P. (1989). Genetics, 122, 19-27.

Tachibana K, Sato T, D'Avirro N and Morimoto C. (1995). J. Exp. Med., 182, 1089-1099.

Templeton DJ. (1992). Mol. Cell. Biol., 12, $435-443$.

Tong X and Howley PM. (1997). Proc. Natl. Acad. Sci. USA, 94, $4412-4417$.

Trujillo JM and Mounts P. (1996). Virology, 220, 1-9.

Turner CE. (1994). Bioessays, 16, 47-52.

Turner CE, Glenney Jr, J and Burridge K. (1990). J. Cell. Biol., 111, 1059-1068.

Turner CE and Miller JT. (1994). J. Cell. Sci., 107, $1583-$ 1591.

Vande Pol S and Howley P. (1994). J. Virol., 69, 395-402.

Walter G, Ruediger R, Slaughter C and Mumby M. (1990). Proc. Natl. Acad. Sci. USA, 87, 2521-2525.

Weng Z, Taylor JA, Turner CE, Brugge JS and Seidel-Dugan C. (1993). J. Biol. Chem., 268, $14956-14963$.

Wood CK, Turner CE, Jackson P and Critchley DR. (1994). J. Cell. Sci., 107, 709-717.

Yan M and Templeton DJ. (1994). J. Biol. Chem., 269, $19067-19073$.

Yang YC, Okayama H and Howley PM. (1985). Proc. Natl. Acad. Sci. USA, 82, 1030-1034. 